\title{
Philosophiques
}

\section{L’agrégation, le programme : la chance de La vie la mort}

\section{Michael Naas}

Volume 47, numéro 2, automne 2020

\section{Derrida en cours}

URI : https://id.erudit.org/iderudit/1075126ar

DOI : https://doi.org/10.7202/1075126ar

Aller au sommaire du numéro

\section{Éditeur(s)}

Société de philosophie du Québec

\section{ISSN}

0316-2923 (imprimé)

1492-1391 (numérique)

Découvrir la revue

\section{Citer cet article}

Naas, M. (2020). L'agrégation, le programme : la chance de La vie la mort. Philosophiques, 47(2), 315-332. https://doi.org/10.7202/1075126ar

\section{Résumé de l'article}

Cet essai porte sur le séminaire La vie la mort (1975-1976) et sur la notion double de "programme " qui l'informe. Dans ce séminaire, Derrida juxtapose en effet une lecture de la notion de " programme ", utilisée par le biologiste français François Jacob dans sa description du fonctionnement de l'ADN, à une réflexion sur le " programme " d'agrégation en France qui avait cette année-là pour thème " Vie et mort ». Cet article commence donc par examiner le rôle crucial joué par l'examen d'agrégation dans la production, voire la reproduction, de la philosophie en tant que discipline en France. Il analyse ensuite la lecture de La logique du vivant que fait Derrida dans ce séminaire à partir de ces deux notions supposément distinctes de " programmes » : un programme soi-disant inflexible et partagé par tous les êtres vivants, celui de l'ADN, et un programme soi-disant plus libre et plus souple, celui de la culture et des institutions humaines comme celles de l'agrégation. En résulte une refonte complète des formes de production et de reproduction en jeu aussi bien du côté de notre compréhension de la génétique que de l'éducation.
Ce document est protégé par la loi sur le droit d'auteur. L’utilisation des services d’Érudit (y compris la reproduction) est assujettie à sa politique d'utilisation que vous pouvez consulter en ligne.

https://apropos.erudit.org/fr/usagers/politique-dutilisation/ 


\title{
L'agrégation, le programme: la chance de La vie la mort
}

\author{
MICHAEL NAAS
}

Département de philosophie, DePaul University (Chicago)

\begin{abstract}
RÉSUMÉ. - Cet essai porte sur le séminaire La vie la mort (1975-1976) et sur la notion double de «programme» qui l'informe. Dans ce séminaire, Derrida juxtapose en effet une lecture de la notion de «programme», utilisée par le biologiste français François Jacob dans sa description du fonctionnement de l'ADN, à une réflexion sur le «programme» d'agrégation en France qui avait cette année-là pour thème «Vie et mort». Cet article commence donc par examiner le rôle crucial joué par l'examen d'agrégation dans la production, voire la reproduction, de la philosophie en tant que discipline en France. II analyse ensuite la lecture de La logique du vivant que fait Derrida dans ce séminaire à partir de ces deux notions supposément distinctes de «programmes »: un programme soidisant inflexible et partagé par tous les êtres vivants, celui de l'ADN, et un programme soi-disant plus libre et plus souple, celui de la culture et des institutions humaines comme celles de l'agrégation. En résulte une refonte complète des formes de production et de reproduction en jeu aussi bien du côté de notre compréhension de la génétique que de l'éducation.
\end{abstract}

\begin{abstract}
This essay focuses on Derrida's 1975-1976 seminar Life Death and the dual notion of "program" that informs it. In this seminar, Derrida juxtaposes a reading of the notion of "program" used by French biologist François Jacob in his depiction of the workings of DNA with reflections on the "program" of the agrégation exam that had set as its topic for that year "Life and Death." The paper thus begins by looking at the critical role played by the agrégation exam in the production or reproduction of philosophy as a discipline in France. It then analyzes Derrida's reading of Jacob's La logique du vivant in terms of two supposedly distinct programs, the putatively inflexible program of DNA, a program shared by all living beings, and the freer, more flexible program of human culture and institutions, including pedagogical institutions such as the agrégation. The result, I argue, is a complete rethinking of the forms of production or reproduction at stake in both genetics and education.
\end{abstract}

Pour Alan Schrift

Mes collègues ont dû vous dire les raisons de leur sévérité pour telles de vos explications [...]. Votre talent n'est aucunement en cause et, comme chaque année - c'est la loi de l'agrégation —, nous avons dû recevoir des candidats d'une «qualité» intellectuelle très inférieure à telles victimes de l'écrit ou de

l'oral, mais qui ont joué le jeu et ont emporté le succès par leur conscience et leur patience. N'oubliez pas que la «leçon » de l'agrégation n'est pas un exercice de virtuosité pure, mais d'abord un travail scolaire, qui doit être assimilable par des élèves - ce qui n'empêche pas qu'une fois dites, rapidement, les choses que vous diriez dans votre classe, vous puissiez parler pour votre jury.

— Maurice de Gandillac «Lettre à Jacques Derrida », le 9 août $1955^{1}$.

1. Lettre de Maurice de Gandillac à Jacques Derrida, le 9 août 1955 ; citée dans Benoît Peeters, Derrida, Paris, Flammarion, 2010, p. 98. 
L'importance de l'agrégation dans le système de l'Éducation nationale en France est bien connue, surtout pour ceux qui ont grandi dans ce système. Et cela est particulièrement vrai en philosophie. En règle générale, on a peu de chance d'obtenir un poste dans l'université française sans être reçu à cet examen, et le classement que l'on obtient par rapport aux autres candidats de la même année va déterminer le genre de poste que l'on recevra de l'Éducation nationale. Bref, l'agrégation détermine les carrières dans l'université pour le meilleur et, le plus souvent, pour le pire, car à peine cinq pour cent des candidats qui passent l'examen chaque année réussissent.

Tout cela est, dis-je, bien connu. Ce qui aura été moins connu, jusqu'à récemment, c'est le rôle que joue l'agrégation dans le choix de ce qui est enseigné dans les cours de philosophie qui préparent les étudiants pour cet examen et, donc, l'effet de cet examen sur les intérêts des philosophes et l'orientation que prend la philosophie en France. Ce qui nous est aussi resté presque totalement inconnu jusqu'à maintenant, c'est l'influence de l'agrégation sur l'enseignement et donc sur le travail et les intérêts philosophiques de Jacques Derrida. L'objectif de cet essai est d'explorer cette influence à la lumière de la publication récente de deux séminaires de Derrida des années 1970, deux séminaires qui montrent à quel point les intérêts philosophiques de Derrida ont été formés par son poste à l'École normale supérieure à cette époque, c'est-à-dire son poste d'agrégé-répétiteur chargé de préparer les étudiants pour cet examen si déterminant.

Mais avant de considérer l'influence de l'agrégation sur Derrida, j'aimerais commencer avec une anecdote afin de remercier ici mon ami Alan Schrift, dont le travail sera essentiel pour ce qui suit, et afin de démontrer comment l'agrégation nous a récemment aidés à comprendre non seulement le contenu des séminaires de Derrida - les figures, les textes, et les thèmes - mais les dates auxquelles ils ont été donnés. Car, si surprenant que cela puisse sembler, quelques-unes de ces dates étaient restées incertaines et il se trouve que c'est l'agrégation qui nous a aidés à les déterminer. Enfin, je commence ainsi parce que c'est une anecdote qui porte sur la chance, sur la relation entre la chance ou la contingence et le déterminisme ou la programmation, notions qui sont au cœur théorique de plusieurs des séminaires de Derrida et qui sont aussi, comme j'essaierai de le suggérer, mises en pratique ou mises à l'épreuve dans la publication et la traduction récentes de ces séminaires.

Voici donc l'anecdote. À Paris pendant l'été 2017, j'ai partagé avec Alan Schrift, ancien professeur de philosophie à Grinnell College qui habite maintenant Paris, une petite incertitude que tous ceux qui travaillent ensemble à la traduction des séminaires de Derrida (les membres du «Derrida Seminar Translation Project $\left.{ }^{2} »\right)$ avaient à propos des dates de deux sémi-

2. Voir la page du «Derrida Seminar Translation Project» [en ligne: http://derridaseminars. org/], consulté le 11 mai 2019. 
naires des années 1970. Quelques mois auparavant, les Éditions Galilée avaient publié le séminaire Théorie et pratique avec le sous-titre Cours de l'ENS-Ulm 1975-1976 et, l'été précédent, c'est-à-dire en 2016, notre équipe de traducteurs s'était réunie afin de travailler sur la traduction anglaise de ce séminaire ${ }^{3}$. Suivant donc l'édition française et toutes les bibliographies disponibles, nous avons nous aussi cru travailler sur un séminaire de 19751976. Et puisque Derrida se réfère par endroits dans Théorie et pratique à son séminaire de "l'année précédente" au sujet de «la vie la mort », nous avons cru (les tapuscrits d'aucun des deux séminaires n'étant datés) que ce séminaire précédent était de 1974-1975, dates qui, une fois de plus, étaient confirmées par toutes les bibliographies que nous avions consultées. Le séminaire La vie la mort, dont Pascale-Anne Brault et Peggy Kamuf étaient en train de préparer l'édition française, datait donc de 1974-1975 et Théorie et pratique de l'année suivante: 1975-1976.

Mais au fur et à mesure que Pascale-Anne Brault et Peggy Kamuf avançaient dans leur travail sur La vie la mort, elles — et à leur suite, tous les membres de l'équipe de l'édition et de la traduction — ont commencé à entretenir des doutes. On avait noté que Derrida se référait lors du séminaire à des textes récemment publiés qui n'étaient en principe pas encore publiés en 1974-1975, et — l'un des exemples les plus intéressants de leur travail de chercheuses détectives - plusieurs séances de La vie la mort avaient été dactylographiées sur le verso de feuilles qui annonçaient pour avril 1975 une réunion (un "meeting de mobilisation») du Groupe de recherches sur l'enseignement philosophique (GREPH) à l'Université de Paris à Jussieu. Il aurait été bien bizarre, avons-nous tous pensé, que Derrida utilise comme papier brouillon en octobre ou en novembre 1974 des photocopies qui annonçaient un événement qui n'aurait lieu que six mois plus tard. Étant donné la nature plutôt improvisée du GREPH, cela aurait été d'autant plus surprenant que l'événement ait été organisé de manière si achevée six mois à l'avance. Nous avons donc commencé à soupçonner, mais sans preuve définitive, que La vie la mort, et non pas Théorie et pratique, avait été donné en 1975-1976, et que ce dernier séminaire avait eu lieu l'année suivante, c'est-à-dire en 1976-1977.

Ce qui trancherait alors la question pour de bon, ai-je dit à Alan après lui avoir raconté cette longue histoire, serait de retrouver les programmes d'agrégation pour ces deux années-là, puisque nous savions que ces séminaires étaient donnés à l'École normale supérieure afin de préparer les étudiants de l'ENS pour cet examen de fin d'année. Ces programmes nous donneraient une preuve définitive, mais, hélas, malgré tous nos efforts à la bibliothèque de l'ENS et à la Bibliothèque nationale, ces documents étaient

3. Jacques Derrida, Théorie et pratique. Cours de l'ENS-Ulm 1975-1976 [sic], Alexander García Düttman (dir.), Paris, Galilée, 2017; Theory and Practice, trad. angl., David Wills, Chicago, University of Chicago Press, 2019. 
restés introuvables. C'est à ce moment-là qu'Alan s'est levé, sans dire un mot, qu'il est monté dans son bureau, et qu'il est revenu deux minutes plus tard avec les photocopies de ces mêmes programmes d'agrégation que nous avions cherchés partout à Paris. Tout était bien là: on pouvait y lire très clairement que le sujet pour la deuxième épreuve de l'agrégation de 1976 était «La vie et la mort» et que le sujet pour la deuxième éprenve en 1977 était, tout aussi clairement, "Théorie et pratique». Comme nous l'avions donc soupçonné, le séminaire La vie la mort, qui préparait les étudiants de l'ENS pour l'examen du printemps 1976, avait été donné au cours de l'année académique 1975-1976, et Théorie et pratique, qui préparait les étudiants pour l'examen du printemps 1977, avait été donné par conséquent en 19761977 et non en 1975-1976 comme la publication récente aux Éditions Galilée le suggérait. C'est ainsi que l'examen de l'agrégation, avec l'aide précieuse d'Alan Schrift, avait été plus utile à la détermination des dates des séminaires de Derrida que tout ce qui se trouvait dans ces séminaires ou dans toutes nos bibliographies.

Mais j'aurais dû le savoir bien en avance - non pas pour ce qui concerne ces dates précises, mais là où il fallait aller pour les confirmer. Car Alan Schrift est, je le savais bien, l'auteur d'un article publié en 2008 dans le Journal of the History of Philosophy sur l'histoire de l'agrégation, et surtout l'agrégation de philosophie depuis son inauguration en 1766 jusqu'en 2008 et, plus intéressant encore, sur "les effets que cette histoire a eus sur les pratiques philosophiques en France ${ }^{4}$ ». Schrift y analyse en détail l'ampleur des effets de cet examen typiquement français et, en particulier, les effets du programme de l'examen, à la fois sur les étudiants qui doivent se préparer pour celui-ci et sur les professeurs qui doivent les préparer. Comme Schrift le fait remarquer, le programme de l'agrégation détermine en très grande partie l'avenir de la philosophie elle-même en France: il détermine ce que les étudiants en philosophie vont étudier, ce que les professeurs vont enseigner, c'est-à-dire les sujets, les figures et les textes qui seront lus, analysés et étudiés dans les cours et les séminaires qui ont pour objet la préparation des étudiants à cet examen. Résultat: il détermine en large mesure ce que ces mêmes professeurs et parfois ces étudiants vont publier dans les mois et les années qui suivront l'examen ${ }^{5}$. De plus, dans la mesure où les professeurs doivent former leurs étudiants non seulement à partir des figures et des textes qu'ils vont enseigner, mais selon un style argumentatif et une rhétorique dont ils estiment que les autorités institutionnelles responsables de la notation trouveront légitimes ou acceptables, ces mêmes professeurs se

4. Alan Schrift, "The Effects of the Agrégation de Philosophie on Twentieth-Century French Philosophy", Journal of the History of Philosophy, vol. 46, n 3, 2008, p. 449. Ma traduction.

5. Ibid., p. 453. 
sentent responsables d'enseigner et donc de renforcer certaines normes ou standards très particuliers de l'écriture philosophique ${ }^{6}$.

L'examen préprogramme ou programme en amont, donc, à la fois les figures et les textes canoniques qui seront enseignés et étudiés - et, même avant cela, quelles figures et quels textes sont considérés comme "canoniques » — ainsi qu'une certaine rhétorique institutionnelle nécessaire pour démontrer ses connaissances. Selon l'analyse de Schrift, l'agrégation est une énorme feedback loop, un système de production autorégulateur ou, plus exactement de reproduction, qui détermine ou bien programme à l'avance ce que la philosophie légitime et compétente était et ce qu'elle sera à l'avenir en France. Il détermine quels philosophes et quelles philosophies seront sélectionnés pour "survivre" dans l'université et lesquels seront laissés de côté par cette sélection? ${ }^{7}$.

Or nous avons appris des biographies de Jacques Derrida, mais aussi de quelques-unes de ses propres publications comme $D u$ droit à la philosophie, que personne ne fut plus conscient que Derrida lui-même des contraintes ou des limitations de ces institutions de reproduction ou d'auto-reproduction dans l'université et plus généralement dans l'Éducation nationale, lui qui, dans son rôle d'agrégé-répétiteur à l'École normale supérieure (qu'on appelle caïman dans l'argot de l'ENS), fut chargé pendant près de vingt ans de préparer les étudiants pour l'agrégation. Derrida, en effet, écrit ceci en 1976 au sujet de son rôle d'agrégé-répétiteur:

Répétiteur, l'agrégé-répétiteur ne devrait rien produire, si du moins produire voulait dire innover, transformer, faire advenir le nouveau. Il est destiné à répéter et à faire répéter, reproduire et faire reproduire: des formes, des normes et un contenu. Il doit assister les élèves dans la lecture et l'intelligence des textes, les aider dans l'interprétation, et à comprendre ce qu'on attend d'eux, ce à quoi ils doivent répondre aux diverses étapes du contrôle et de la sélection, du point de vue des contenus ou de l'organisation logico-rhétorique de leurs exercices (explications de texte, dissertations ou leçons). Il doit donc se faire auprès des élèves le représentant d'un système de reproduction $[\ldots]$ ou plutôt l'expert qui, passant pour mieux connaître la demande à laquelle il a dû d'abord se plier, l'explique, la traduit, la répète et re-présente donc pour les jeunes aspirants ${ }^{8}$.

6. Ibid., p. 453, n. 14.

7. Schrift écrit: «Quand un texte philosophique apparait sur le Programme pour l'examen oral de l'agrégation, cela veut dire que tous les étudiants de cette année aspirant à une carrière en philosophie vont passer l'année à lire ce texte très attentivement » (ibid., p. 453). Ma traduction.

8. Derrida, "Où commence et comment finit un corps enseignant», dans Du droit à la philosophie, Paris, Galilée, 1990, p. 122. Ce texte a paru d'abord dans l'œuvre collective Politiques de la philosophie (Paris, Grasset, 1976) aux côtés de textes de Gilles Châtelet, Michel Foucault, Jean-François Lyotard et Michel Serres. 
On entend bien dans ce texte de 1976 l'ambivalence, sinon les objections, de Derrida à l'égard du système de l'agrégation et du rôle qu'il a lui-même joué dans ce système. Mais avec la publication assez récente des séminaires de Derrida, nous voyons que cette ambivalence et ces objections se faisaient entendre aussi dans les séminaires eux-mêmes qui avaient pour but la préparation des étudiants pour l'agrégation. Cela est particulièrement clair dans les deux séminaires que j'ai évoqués ci-dessus, c'est-à-dire dans La vie la mort (1975-1976) et Théorie et pratique (1976-1977). Nous entendons Derrida au début de chacun de ces deux séminaires se demander, à haute voix et devant ses étudiants, comment il va à la fois respecter son obligation d'aider ses étudiants à se préparer pour l'examen qui déterminera toute leur carrière en philosophie et marquer son grand mécontentement envers tout ce système agrégatif. Comment, en un mot, va-t-il «négocier» entre des obligations si diverses et parfois même antithétiques? Comment va-t-il préparer ses étudiants pour l'examen et donner un séminaire - comme Derrida aimait le dire — «digne de ce nom»?

Derrida commence son séminaire de 1975-1976 en expliquant le titre qu'il lui a donné, un titre qui suggère qu'il s'agira bien de la vie et de la mort, les deux sujets annoncés pour l'agrégation de cette année-là, mais pas exactement comme le programme l'avait prescrit, à savoir «la vie et la mort». C'est une petite différence qui change presque tout. Derrida dit au début de la première séance du séminaire dont le titre, annoncé par avance est, je le rappelle, La vie la mort:

La suspension du et dans mon titre, dans le titre du séminaire constitue une intervention bien discrète, fort peu violente, direz-vous, dans le programme d'agrégation qui porte, cette année, très traditionnellement, $<$ le titre $>$ « La vie et la mort", la vie d'abord et la mort ensuite. Tous les ans, depuis quelques années, au début de chaque séminaire, certains d'entre vous le savent, je m'explique sur le malaise où je suis de me régler dans ce travail, ici, sur le programme d'agrégation et sur la décision stratégique que je prends, encore une fois, tout en luttant contre l'institution agrégative, ailleurs et ici même, de négocier avec elle dans des conditions données. Je ne vais pas répéter ce que j'ai dit déjà et reproduire indéfiniment le même schéma. Je voudrais plutôt, en analysant l'intitulé du programme d'agrégation, non pas m'y conformer, mais en faire l'objet - à déconstruire - de ce séminaire?

Derrida dit cela juste après avoir parlé assez longuement de sa déformation du sujet donné par l'agrégation, c'est-à-dire après avoir expliqué que son sujet sera «La vie la mort» et non, comme le comité de l'agrégation l'avait prescrit, "La vie et la mort», ou bien, si on entend le mot entre «la vie» et «la mort» non comme et mais comme est, «La vie est la mort», comme un certain Hegel l'aurait soutenu, la vie comme la vérité de la mort ou la vie

9. Derrida, La vie la mort. Séminaire (1975-1976), Pascale-Anne Brault et Peggy Kamuf (dir.), Paris, Seuil, 2019, p. 25. 
spirituelle comme ce qui relève la vie biologique, etc. Si le programme de l'agrégation dit "La vie et la mort ", Derrida essaiera pour sa part - et cela sera son compromis, sa négociation - de penser "La vie la mort", une autre logique de la vie et de la mort qui sera développée au cours des quelque quatorze séances à partir de patientes lectures de Nietzsche, de Heidegger, de Freud et, le plus surprenant, de François Jacob, médecin et biologiste, lauréat du Prix Nobel en 1965 pour ses travaux en génétique. C’est chez ce dernier que Derrida va trouver, afin de la questionner - et nous allons le suivre en détail dans un instant - l'opposition entre un programme (c'est le mot de Jacob) génétique, le programme de l'ADN qui commande la reproduction de tout être vivant, et un programme humain, institutionnel, cérébral, culturel, etc., programme qui inclut, il va sans dire, toutes les institutions d'apprentissage et de pédagogie. C'est une lecture importante pour penser les implications de la déconstruction pour les sciences naturelles aussi bien que les implications des sciences naturelles pour la déconstruction. Mais Derrida ne tardera pas à mettre en rapport ces deux sortes de programmes et le genre de reproduction que chacun implique avec le programme de l'agrégation elle-même, afin de suggérer que ce dernier est, en fin de compte, un système de reproduction qui n'est pas moins rigide que celui de l'ADN (selon Jacob) et que l'optimisme de Jacob à l'égard de la promesse du programme culturel et institutionnel n'est pas aussi légitime ou justifié qu'il ne le croit.

Alors, ce système de reproduction pédagogique est bien en évidence, comme nous l'avons suggéré ci-dessus, non seulement dans des figures, des textes et des sujets auxquels l'agrégation donne la priorité, mais aussi dans le style et la rhétorique qui seront évalués positivement par le jury d'agrégation. À la fin de la première séance de La vie la mort, Derrida revient donc encore une fois sur le sujet de l'agrégation, cette fois-ci moins pour parler du «contenu» de l'examen, des sujets qu'il faut aborder, mais plutôt du style avec lequel il faudrait les aborder. Encore une fois, il fait bien entendre son scepticisme, sinon son mécontentement ou son agacement. Ayant déjà évoqué un essai de Georges Canguilhem au sujet de la vie, essai publié sous le titre «Le concept et la vie ${ }^{10}$ ", Derrida semble d'abord faire une vraie concession à l'agrégation en recommandant sérieusement et de bonne foi à ses étudiants de suivre l'exemple de Canguilhem pour l'agrégation: "Je vous en recommande la lecture ainsi que de tout l'ouvrage [où il apparaît], ainsi que de $L a$ connaissance de la vie, ouvrage antérieur. Tout cela vous sera très utile du point de vue de l'agrégation ${ }^{11}$. » Mais, après avoir cité un bref passage de cet essai de Canguilhem, auquel il va revenir dans les séances ultérieures,

10. Georges Canguilhem, «Le concept et la vie», Revue philosophique de Louvain, vol. 64, no 82,1966 , p. 193-223.

11. Derrida, La vie la mort, p. 41. Voir Canguilhem, La connaissance de la vie, Paris, Vrin, 1965. 
Derrida laisse peu de doute — et, dans l'analyse qui suit, aucun doute — sur son réel sentiment quant à ce genre de «rhétorique agrégative» à l'œuvre chez Canguilhem:

C'est pour y prendre aussi des modèles de rhétorique agrégative que je vous conseillais de lire Canguilhem, et pour y suivre, comme chez tous les épistémologues de l'école française, des effets de l'agrégation, de l'école française ${ }^{12}$.

On retrouve ce même geste au début du séminaire Théorie et pratique, un séminaire donné, on s'en souvient, pour préparer les étudiants à une agrégation où il fallait traiter du sujet "Théorie et pratique». Au lieu de déformer le titre du programme de l'agrégation comme il l'avait fait l'année précédente, Derrida décide cette fois-ci de garder le titre comme tel, mais non sans se livrer à un gros soupir d'exaspération à son sujet. Voici comment il commence ce séminaire de 1976-1977:

Théorie et pratique, donc.

Faut le faire.

Quand je dis «faut le faire», qu'est-ce que je fais?

Bien sûr, apparemment, je pousse un soupir de découragement, teinté de protestation ironique devant le programme qui nous impose de traiter en un an, et sous la forme d'un séminaire, d'une telle question, si c'en est une. J'amorce, comme tous les ans - mais rassurez-vous, je n'irai pas au-delà cette année -, l'analyse critique de la situation qui nous est imposée en vous invitant à ne pas vous contenter de critiquer - en théorie - mais à tenter de transformer effectivement, pratiquement cette situation ${ }^{13}$.

Encore une fois donc, Derrida exprime son mécontentement devant ce système qui impose un tel programme qui détermine dans une large mesure non seulement le contenu mais aussi la forme du séminaire qu'il aura à donner.

Mais revenons à La vie la mort, où Derrida développe sa pensée la plus radicale quant à la relation entre la vie et la mort dans la tradition philosophique occidentale. On y voit à l'œuvre une analyse qui est intéressante et importante en elle-même, mais qui nous enseigne aussi quelque chose de crucial à propos de la lecture ou de la stratégie argumentative de Derrida plus généralement. Avec des lectures de Hegel, de Nietzsche, de Heidegger, de Freud et de François Jacob, Derrida va développer une logique de la vie-la mort qui conteste, comme je l'ai suggéré plus haut, à la fois une logique philosophique de l'opposition (la vie et la mort) et une logique de la subsomption (la vie est la mort). Mais ce qui est aussi significatif dans ce séminaire, bien que moins évident au premier abord, c'est la tentative de Derrida de repenser des catégories ou des oppositions philosophiques telles

12. Ibid., p. 42.

13. Derrida, Théorie et pratique, p. 13. 
que téléologie et contingence, nécessité et chance, ou déterminisme et liberté, autour de la question du programme justement. Cela n'est jamais plus clair que dans la lecture que Derrida nous propose de Jacob. Celui-ci consacre en effet trois séances consécutives - la quatrième séance jusqu'à la sixième, ainsi qu'une grande partie de la première (plus d'une centaine de pages tapuscrites en tout) - à La logique du vivant de Jacob, un livre de 1970 qui essaie d'expliquer la reproduction des êtres vivants en termes de code génétique ou de programme de l'ADN ${ }^{14}$.

C'est cette notion de programme, en effet, qui va expliquer pourquoi Derrida estime que La logique du vivant de François Jacob, un livre écrit par un biologiste généticien et non par un philosophe, est un objet de critique philosophique légitime. Le problème résidera moins dans le fait que Jacob lui-même utilise un langage philosophique pour décrire et interpréter son propre projet mais plutôt dans le fait qu'il affirmera dans La logique $d u$ vivant qu'en parlant de "vivants» et non pas de «la vie », il est capable d'éviter toute l'architecture et tous les faux problèmes de la métaphysique occidentale, toutes les conceptions vagues mais tenaces de la tradition métaphysique et théologique. Derrida va vite faire entendre qu'il ne croit rien de ces revendications de Jacob qui, malgré toutes ses protestations au contraire, répète, reproduit et réinscrit à l'intérieur de son discours des notions de métaphysique traditionnelle telles que la téléologie, l'essence et, surtout, la vie. Derrida va donc démontrer non seulement que Jacob n'a pas évité une telle métaphysique, mais qu'il s'est fait piéger par quelques aspects les plus traditionnels de cette métaphysique en réinscrivant une notion de téléologie dans sa conception des êtres vivants et en projetant un humanisme problématique dans sa hiérarchisation de ceux-ci.

C'est toujours dans la première séance de La vie la mort, après avoir parlé de l'agrégation et de son programme pour l'année, que Derrida entame sa lecture de Jacob. Il commence en allant droit au but, c'est-à-dire droit à la notion de "programme" dans La logique du vivant. Mais il amorce sa critique de Jacob en se référant — chose assez rare dans les séminaires — à son propre usage de cette idée de "programme» dans une publication antérieure, à savoir dans De la grammatologie. Derrida s'y réfère et même se cite:

Il y a quelque dix ans, dans De la grammatologie, un chapitre près du début qui s'intitulait (déjà, dirait-on, par coïncidence, prescience ou téléologie presque sans sujet) «Le programme», je rappelais que, je cite, «le biologiste parle aujourd'hui d'écriture et de pro-gramme à propos des processus les plus

14. François Jacob, La logique du vivant. Une histoire de l'hérédité, Paris, Gallimard, 1970. Pour une lecture brillante du projet de Derrida au sujet des "sciences de la vie ", depuis "Freud et la scène de l'écriture » et De la grammatologie jusqu'au séminaire La vie la mort et les œuvres plus tardives sur l'auto-immunité, voir Francesco Vitale, Biodeconstruction, trad. angl., Mauro Senatore, Albany, New York, State University of New York Press, 2018. 
élémentaires de l'information dans la cellule vivante ${ }^{15} »$. Mais ce n'était pas pour réinvestir dans la notion ou le mot de "programme» toute la machine conceptuelle du logos et de sa sémantique, mais pour tenter de montrer que l'appel à une écriture non phonétique dans la génétique devait, devrait impliquer et provoquer toute une déconstruction de la machine logocentrique plutôt que de provoquer un retour à Aristote ${ }^{16}$.

Derrida arrive à cette conclusion critique au cours d'une longue analyse de la notion de programme chez Jacob, notion qui est essentielle dans La logique du vivant. Jacob y distingue alors entre deux programmes ou deux sortes de programmes, chacun avec sa propre forme de mémoire. Le premier, c'est le programme génétique, le programme de l'ADN que nous, êtres humains, partageons avec tout autre être vivant. Jacob qualifie ce programme de rigide et d'inflexible, avec une forme de mémoire qui marche par la simple répétition ou la re-transcription d'un code préétabli. C'est la mémoire codée de l'acide désoxyribonucléique, mémoire dans la forme d'un code génétique qui se transmet inflexiblement d'une génération à l'autre.

L'autre programme, qu'il faut donc distinguer rigoureusement de ce premier, selon Jacob, c'est le programme institutionnel, culturel, cérébral, mental (ou nerveux), de l'humain, le programme qui sépare en effet les êtres humains de tout autre être vivant. La mémoire de ce programme-ci est, selon Jacob, beaucoup plus flexible que celle du programme génétique, capable d'être transformée dans la mesure où elle reçoit des leçons de l'expérience et peut donc transmettre de l'information acquise par cette expérience d'une génération à l'autre.

Il y a donc deux programmes, l'un inflexible et incapable de recevoir des leçons de l'expérience, l'autre flexible et capable de transmettre des acquis d'une génération à la suivante. Derrida résume:

On devrait donc en conclure que le programme nerveux (institutionnel) a rapport au dehors, reçoit les leçons de l'expérience, se laisse transformer, alors que le programme génétique forme un système clos et sourd, purement endogène, imperméable au changement que Jacob appelle d'un mot fort suspect "délibéré ${ }^{17} »$.

Or Derrida ici soupçonne déjà l'usage que Jacob fait des termes et des catégories philosophiques héritières d'une longue et lourde tradition. Selon Jacob, le programme institutionnel est capable de changer par le biais des interventions "délibérées", c’est-à-dire libres, conscientes et venues du dehors, tandis que le programme génétique est fermé, "sourd » dit Jacob, à tout ce qui vient de l'extérieur et ne peut donc subir aucune intervention délibérée. Le programme génétique peut changer, bien sûr, par le biais de

15. Jacques Derrida, De la grammatologie, Paris, Minuit, 1967, p. 19.

16. Derrida, La vie la mort, p. 44.

17. Ibid., p. 38-39. 
mutations, mais comme Derrida le souligne avec les mots de Jacob: «[E]n aucun cas il ne peut y avoir de corrélation entre la cause et l'effet de la mutation». Bref, "pour chaque individu le programme résulte d'une cascade d'évènements, tous contingents" et "la nature même du code génétique empêche tout changement délibéré du programme sous l'effet de son action ou du milieu ${ }^{18}$. À la différence, donc, du programme institutionnel et cérébral, le programme génétique "ne reçoit pas les leçons de l'expérience ${ }^{19}$ ", bien qu'il change par des mutations qui sont - et l'analogie vient ici de Jacob - comme les erreurs non intentionnelles d'un copiste ou d'un imprimeur, des erreurs qui, une fois introduites, sont transmises fidèlement d'une génération à l'autre. Pour suivre l'analogie, si le programme génétique change à cause des erreurs non intentionnelles d'un copiste ou d'un imprimeur, le programme institutionnel est capable de changer selon les projets ou les desseins conscients, intentionnels ou délibérés d'un programmeur ou, mieux, d'un dirigeant qui exécute ses projets par la parole.

Le programme génétique est donc caractérisé par la contingence, par des causes qui viennent sous forme de mutations complètement non planifiées, imprévisibles, non délibérées, contingentes. Notons donc que la contingence est opposée par Jacob non pas à la nécessité, puisque le programme génétique suit une chaîne de causes et d'effets plus ou moins nécessaire, mais à la liberté, à la délibération, à la conscience, etc. Le programme génétique est ainsi associé par Jacob avec tout ce qui est typiquement attaché à la contingence et avec l'extériorité dans la tradition métaphysique, à savoir avec une certaine automaticité, avec des signifiants sevrés de signifiés, des signes sevrés de leur sens, etc. Au premier abord, tout cela semble indiquer une énorme rupture avec la tradition métaphysique puisque Jacob identifie la vie elle-même - ou au moins le code ou le programme des êtres vivants, les mécanismes de l'ADN - avec la contingence ou la chance. Là où les philosophes et les théologiens auront par le passé trouvé ou projeté un logos ordonné et ordonnant, une téléologie qui mène à l'humain, quelquefois même un souffle créateur, Jacob aura placé ou projeté - selon les apparences - une certaine contingence, une sorte de chance aveugle. C'est bien, semble-t-il, une interruption, sinon une déconstruction, de la tradition métaphysique.

Mais retournons un instant à l'autre programme, c'est-à-dire, au programme institutionnel ou cérébral. C'est le programme par lequel, selon le schéma de Jacob, l'information est transmise d'une génération humaine à la suivante non pas de façon contingente, non intentionnelle, aveugle, comme c'est le cas pour le programme génétique, mais de façon libre, intentionnelle ou délibérée par la culture et les institutions humaines. Ce programme s'est développé grâce à une mutation dans le programme génétique, bien sûr,

18. Jacob, La logique du vivant, p. 11; cité par Derrida dans La vie la mort, p. 39. 19. Ibid. 
mais cette mutation fut si radicale que le résultat a été une forme complètement nouvelle de mémoire et de programme. Elle fut si radicale que le programme cérébral a pu se libérer de la rigidité du programme génétique lui-même par les institutions qui peuvent, de façon délibérée, transmettre sélectivement de l'information d'une génération à l'autre. Plus radicale encore, avec la découverte des mécanismes de l'ADN au milieu du vingtième siècle, il est enfin devenu possible d'intervenir dans la transcription auparavant aveugle ou sourde de l'ADN, c'est-à-dire dans le processus même de l'hérédité, afin de manipuler et de changer le programme génétique de l'homme et des autres êtres vivants. Tandis qu'il y avait toujours des changements dans le processus d'évolution sous forme de mutations, celles-ci se produisaient entièrement par "chance ", c'est-à-dire sans aucune délibération ou dessein, aucune corrélation entre les causes et les effets. Le programme institutionnel aura été donc le "supplément» — c'est le mot de Jacob - au programme génétique qui permet à l'homme aujourd'hui de modifier le programme génétique lui-même, en remplaçant la chance ou la contingence par la planification humaine, libre et délibérée. Voici comment Jacob, près de la fin de La logique du vivant, imagine la possibilité pour l'homme de contrôler l'évolution à travers le programme génétique:

Avec l'accumulation de la connaissance, l'homme est devenu le premier produit de l'évolution capable de maîtriser l'évolution. [...] Peut-être un jour pourra-t-on intervenir sur l'exécution du programme génétique, voire sur sa structure, pour en corriger certains défauts, pour y glisser des suppléments. Peut-être aussi parviendra-t-on à produire à volonté, en autant d'exemplaires que désiré, la copie exacte d'un individu, un homme politique, un artiste, une reine de beauté, un athlète par exemple. Rien n'empêche d'appliquer dès maintenant aux êtres humains les procédés de sélection utilisés pour les chevaux de course, les souris de laboratoire ou les vaches laitières ${ }^{20}$.

Jacob peut donc affirmer que le génie génétique aura, en effet, résolu le long débat en biologie sur la question de savoir si les "caractères acquis " peuvent être hérités. Le programme cérébral et institutionnel permet à l'homme non seulement de recevoir des leçons de l'expérience et de les transmettre à des générations suivantes mais de modifier certains traits de son propre ADN et de les transmettre aux générations futures. Comme on vient de lire dans les dernières lignes de La logique du vivant, Jacob n'est pas simplement enthousiaste mais exubérant à l'idée de cette possibilité. Bien évidemment, il n'est pas nécessaire de chercher trop loin dans le vingtième siècle pour voir comment ce rêve de contrôler le processus d'évolution, le rêve d'exclure par sélection certaines maladies néfastes des hommes et des animaux, peut devenir un cauchemar eugénique. Cette critique peut bien s'entendre dans l'analyse de Derrida, mais elle est loin d'être la seule à être développée. 
Derrida va, en effet, remettre en question l'opposition elle-même entre le programme génétique et le programme institutionnel, c'est-à-dire l'opposition entre un programme prétendument rigide, inflexible, exposé à la contingence et un programme délibéré, contrôlé ou au moins contrôlable par la planification humaine. Il commence cette remise en question - selon une stratégie, une logique, que l'on retrouve à l'œuvre dans presque tous les textes de Derrida, du début jusqu'à la fin - en demandant si le côté de l'opposition qui a le moins de valeur, qui aura été traditionnellement dénigré ou réprimé, peut être si facilement séparé du "bon" côté, le côté qui est censé avoir plus de valeur. Dans ce cas particulier, il commence en demandant si le programme institutionnel, le programme humain, le programme qui promet le contrôle, la manipulation, la transformation du programme génétique de façon délibérée, est bien aussi délibéré qu'on le croit, s'il n'y a pas une sorte de contingence également à l'œuvre dans celui-ci. Ayant cité Jacob sur l'opposition entre ces deux programmes, Derrida constate:

Pour que, ainsi décrit, le programme génétique s'oppose de façon pertinente au programme nerveux, cérébralo-institutionnel, il faudrait être assuré qu'on ne puisse pas dire la même chose de celui-ci. Or ne peut-on pas dire la même chose de lui ${ }^{21}$ ?

Il ajoute immédiatement après - et ceci, il faut le rappeler, a été écrit en 1975-1976:

S'il est un acquis général d'un certain nombre de ruptures théoriques dans ce que j'appelle pour faire vite, pour l'instant, la modernité, c'est que la causalité dans l'ordre des programmes disons «cérébraux-institutionnels» (psychiques, sociaux, culturels, institutionnels, politico-économiques, etc.), cette causalité a exactement le style, dans ses lois, de celle que Jacob semble vouloir réserver aux programmes génétiques, à savoir que, [et Derrida cite ici les mots de Jacob qui portent sur le programme génétique mais en les appliquant ici au programme institutionnel] «tous les phénomènes qui contribuent à la variation des organismes et des populations se produisent en toute ignorance de leurs effets ». [Derrida donc continue après sa citation de Jacob:] De même l'hétérogénéité des causes et des effets, le caractère non délibéré des changements de programme, en un mot tout ce qui place les sujets du dedans du système en situation d'effets inconscients de la causalité, tout ce qui produit des effets de contingence entre l'action venue du dehors et les transformations internes du système, tout cela caractérise aussi bien le programme non génétique que le programme génétique ${ }^{22}$.

Derrida suggère donc que, malgré toute la planification prétendue délibérée, toute la soi-disant liberté qui opère dans le programme institutionnel, cérébral, il y a aussi - comme nous l'avons appris de la psychanalyse, de la

21. Derrida, La vie la mort, p. 40.

22. Ibid. 
sociologie, de l'économie, etc. — toutes sortes d'effets contingents (toujours dans le lexique de Jacob lui-même), c'est-à-dire, des effets non délibérés, inconscients, qui viennent de l'extérieur du programme et qui occasionnent ou bien une répétition du programme ou une transformation selon des processus qui sont hors du contrôle de ses acteurs. Derrida n'est pas en train de nier de façon intégrale, bien sûr, le rôle joué par la conscience, l'intention, la délibération, etc., sur le programme institutionnel ou cérébral. Il est simplement en train de suggérer que ce programme ne peut jamais exclure la part jouée par des effets non conscients, non planifiés, contingents, etc. Et puisque ces effets ne peuvent jamais être exclus, le programme institutionnel est dès le début contaminé par ces effets, son contrôle ou sa maîtrise entamée par eux.

Derrida fait ainsi appel à certaines idées issues des sciences sociales afin de remettre en question les revendications de Jacob au sujet de la noncontingence du programme institutionnel. Il ne lui reste plus qu'à utiliser les acquis de la philosophie afin de diagnostiquer et de critiquer la raison de telles revendications chez Jacob. Je poursuis la citation:

[Mais] où Jacob prend-il qu'en dehors du système et des programmes génétiques le changement du programme est délibéré, essentiellement délibéré? Où le prend-il sinon dans une opposition idéologico-métaphysique qui détermine les programmes supérieurs ou symboliques (avec l'humanité au plus haut d'eux-mêmes) à partir du sens, de la conscience, de la liberté, du savoir de la limite entre le dedans et le dehors, l'objectivité et la non-objectivité, etc. ${ }^{23}$ ?

Ayant suggéré que le programme institutionnel a aussi une part de contingence ou de non-délibéré, Derrida soutient maintenant qu'il y a, chez Jacob, un humanisme métaphysique latent ou implicite dans ce même programme. C'est en effet cet humanisme qui a poussé Jacob à opposer le programme cérébral et le programme génétique sur la base de critères tels que la délibération et la liberté - critères envers lesquels, il faut le noter, Derrida restera sceptique dans tout son travail, les déployant rarement et presque toujours stratégiquement afin d'y opposer ce qui est rigidement déterminé ou prédéterminé24.

Dans son séminaire La vie la mort, Derrida remet en question la revendication de Jacob voulant que le programme institutionnel change ou se

\section{Ibid.}

24. Dans un entretien avec Élisabeth Roudinesco publié en 2001 dans De quoi demain..., Derrida dit dans le contexte d'une discussion qui porte sur la science et - aucune coïncidence ici - le génie ou l'ingénierie génétique: "Je me sers rarement du mot de liberté [...]. En certaines occasions, je défendrai pourtant la liberté comme un excès de complexité par rapport à un état machinal déterminé, et je me battrai pour des libertés, mais je ne parlerai pas tranquillement de la liberté [...], ce mot me paraît souvent chargé de présupposés métaphysiques qui confèrent au sujet ou à la conscience — c'est-à-dire à un sujet égologique — une indépendance souveraine par rapport aux pulsions, au calcul, à l'économie, à la machine ", Jacques Derrida et Élisabeth Roudinesco, De quoi demain..., Paris, Fayard et Galilée, 2001, p. 85-86. 
modifie à cause de décisions libres, délibérées, conscientes de la part de ses acteurs. Selon Derrida, le programme institutionnel change ou subit des mutations non pas seulement par les décisions des acteurs rationnels mais par des causes non délibérées, inconscientes, contingentes, dans le langage de Jacob, causes qui sont plus comme des mutations de l'ADN. S'emparant des termes et des caractéristiques du programme génétique — l'inconscience, la non-délibération, le manque de liberté, la contingence, etc. - et les appliquant au programme institutionnel, Derrida suggère, en effet, que l'humain n'est pas vraiment, malgré toutes les apparences, le maître absolu de son propre programme, c'est-à-dire, le maître du programme cérébral et institutionnel. Cela ne veut pas dire, comme je l'ai suggéré plus haut, qu'il faut complètement abandonner des notions telles que la liberté, la délibération, la conscience, l'intention, etc., mais qu'il faut les utiliser avec beaucoup plus de prudence que ne le fait Jacob. Cela implique aussi que le programme institutionnel ne peut pas être rigoureusement distingué du code génétique sur la base de ces notions. La distinction ou, plutôt, l'opposition chez Jacob entre les deux programmes, l'un marqué par la contingence et l'autre par la non-contingence, semble être elle-même conditionnée par une sorte de contingence généralisée qui marque et détermine, de façons différentes et à des degrés variés, les deux programmes. Derrida conclut ce passage:

Là encore, vous le voyez, l'opposition entre les deux programmes ne peut pas être rigoureuse, et cela me paraît tenir à ce que, faute d'avoir réélaboré et la notion générale de programme et la valeur d'analogie, on les laisse marqués par une téléologie logocentrique et par une sémantique humaniste, par ce que j'appellerai une philosophie de la vie ${ }^{25}$.

Mais en parlant ici d'une "téléologie logocentrique» et d'une "philosophie de la vie ", Derrida semble suggérer encore autre chose. Ce que Jacob dit à propos du programme génétique, ce programme de la contingence, est contredit, semble-t-il, par l'idée d'une certaine téléologie à l'intérieur de ce programme aussi. Cette téléologie ne correspond pas, bien sûr, à quelque projet divin ou dessein intelligent mais à une sorte de logos qui se développe dans le temps, qui se déploie ou se déplie dans l'histoire. Après avoir suggéré que le programme institutionnel est lui-même conditionné par des éléments non délibérés, inconscients, contingents — tous ces éléments que Jacob avait attribués uniquement au programme génétique — Derrida suggère maintenant qu'il y a une téléologie cachée ou subreptice dans l'interprétation que donne Jacob lui-même du programme génétique. Tandis que l'interprétation de Jacob à l'égard de l'ADN en tant que code avait l'air de rompre radicalement avec la tradition métaphysique, elle s'avérerait, en fin de compte, complètement solidaire de cette tradition dans la mesure où ce code est compris de façon logocentrique, c'est-à-dire en termes d'un logos qui développe ou 
d'un dessein prédéterminé qui se déploie dans l'histoire. Si Jacob avait interprété ou compris le code génétique d'un point de vue non logocentrique, son interprétation de la façon dont l'ADN se transmet aurait été très différente, encore plus radicale, et, semble-t-il, plus en accord avec ce que nous avons appris dans les cinquante dernières années par les découvertes, par exemple, de l'épigénétique.

Derrida suggère en effet que, même à l'intérieur du programme génétique, il n'y aurait pour Jacob aucune vraie chance ou contingence dans la mesure où cette téléologie subreptice avait guidé son devenir depuis le début, un développement qui aboutit en fin de compte à l'homme. Derrida essaie dans ces pages du séminaire consacrées à Jacob d'exposer une téléologie et un humanisme latents ou cachés dans l'interprétation du biologiste afin de restaurer à l'analyse un élément de chance ou de contingence, non seulement dans le programme génétique dont les mécanismes furent d'abord révélés et explicités par Jacob et d'autres, mais en particulier dans le programme humain dont Jacob croit l'homme capable d'un contrôle libre, intentionnel, et délibéré.

En fin de compte, ce que Derrida trouve subrepticement à l'œuvre chez Jacob n'est pas si différent de ce qu'il décèle chez beaucoup de philosophes et dans beaucoup de textes philosophiques. Par exemple, ce n'est pas si différent de ce qu'il critique dans la théorie des speech acts de John Austin, à savoir ce que Derrida nomme dans «Signature événement contexte» «le leurre téléologique de la conscience ${ }^{26} »$. Ce leurre ou cette illusion, ce "phantasme ", comme Derrida l'appellera dans des textes ultérieurs, est inhérent, semble-t-il, à la notion même de "programme» telle que Jacob la comprend. Du côté du programme institutionnel, c'est précisément ce "leurre téléologique de la conscience " qui est à l'œuvre, le leurre de la conscience pleine, libre, présente-à-soi. Mais dans le programme génétique, il y a aussi quelque chose qui ressemble à un leurre téléologique du sens, le leurre d'un sens ou d'une signification qui se déplie ou se déploie dans l'histoire humaine et dans l'histoire des êtres vivants en général. Ce sont, dans les deux cas, des aspects de ce que Derrida appelle «logocentrisme», et ils sont présents non seulement chez un penseur comme Husserl qui appartient à la tradition phénoménologique ou chez un philosophe du langage ordinaire comme Austin, mais aussi dans le discours d'un biologiste comme François Jacob.

Le programme institutionnel, cérébral, mental, n'est donc pas aussi conscient ou délibéré que Jacob ne le pense, et Jacob pense comme il le fait, en opposant le programme institutionnel et le programme génétique, en raison d'un humanisme métaphysique qui l'incite à attribuer la liberté et la délibération au programme institutionnel, d'une part, et un logos téléologique au programme génétique de l'autre. Tandis qu'une certaine contin-

26. Jacques Derrida, «Signature événement contexte», dans Marges de la philosophie, Paris, Minuit, 1972, p. 389. 
gence ou une certaine relation au dehors conditionne, selon Derrida, et le programme génétique et le programme institutionnel, ce qui fait qu'il est impossible de distinguer les deux en se référant à des attributs tels que la contingence, d'un côté, et la liberté, de l'autre, Jacob a construit les deux programmes à partir de prémisses humanistes et téléologiques qui, selon lui, ne caractérisent que le programme institutionnel.

Comme je l'ai déjà suggéré, cette analyse de Jacob est intéressante et importante en soi, mais elle nous dit aussi quelque chose de la stratégie générale de Derrida. En localisant ce qui aura été exclu ou marginalisé dans un discours - la contingence, par exemple, sous la forme d'une "cascade d'évènements, tous contingents" - , Derrida peut montrer comment la contingence est à l'œuvre dans tout ce qui a de la valeur, à savoir ici la conscience, la liberté, la délibération, etc. Au lieu d'une opposition entre les deux programmes, définis en termes de qualités telles que la rigidité, l'inflexibilité, la contingence, d'un côté, et la flexibilité, la conscience, la liberté, de l'autre, Derrida suggère que les deux côtés sont des effets d'une contingence générale qui n'est pas sans rapport avec ce que Derrida appelait, dans plusieurs textes des années 1960 et 1970, tels que De la grammatologie et «Signature événement contexte » pour n'en citer que deux, une «itérabilité » ou «citationnalité » générale.

Le résultat de l'analyse de Derrida n'est surtout pas de tout attribuer à la chance ou à la contingence, de dénier le rôle joué par la conscience, la délibération ou la liberté. C'est plutôt de montrer que la part jouée par la chance ne peut jamais être exclue des affaires humaines, que ce qui excède ou qui échappe au programme ne peut pas être exclu de principe par le programme. L'important ici, pour nous, c'est de montrer comment, chaque fois - et chaque fois de façon unique - le non-programmable peut toujours venir interrompre le programme, de façon imprévisible, jamais complètement la même ${ }^{27}$. L'important, c'est de montrer que l'improgrammable n'est pas simplement ce qui arrive au programme comme un accident, mais ce qui définit le programme dès le début, autrement dit, qu'une certaine chance va continuer à hanter les meilleurs projets et les meilleurs programmes - voire tous les projets et les programmes - des souris de laboratoire, des vaches laitières et, bien sûr, des hommes. Le programme de l'agrégation n'échappera donc nullement à cette loi.

Comment, donc, cette analyse des deux programmes chez Jacob peut-elle nous aider à repenser cette notion de programme avec laquelle j'ai commencé, c'est-à-dire, le programme de l'agrégation? Comment l'ana-

27. Il y a, je crois, des thèses à écrire sur la relation entre l'improgrammable et le programme, l'événement, qui est toujours l'imprévisible et non programmable, et la programmabilité de la machine qui neutralise l'événement. Et ces thèses seront longues parce que cette notion de programme ne se trouve pas seulement dans De la grammatologie et La vie la mort, mais dans de nombreux autres textes de Derrida. 
lyse derridienne de cette relation entre les deux programmes, peut-elle nous aider à repenser un "programme » institutionnel tel que l'agrégation? Dans la mesure où l'analyse de Derrida nous montre que le programme institutionnel est beaucoup moins flexible que Jacob ne le croit, moins sous le contrôle de ses acteurs humains, on peut commencer à comprendre l'agrégation et le "programme» qui y est associé comme un énorme système autorégulateur, une énorme feedback loop. On peut enfin commencer à interpréter le programme agrégatif comme un système qui se reproduit avec une efficacité semblable à celle qui est à l'œuvre dans le programme génétique - un énorme système auto-productif où rien ou très peu ne change d'une génération à l'autre, où on lit les mêmes textes de la même façon que nos " ancêtres " philosophiques ${ }^{28}$. C'est comme si ces deux programmes - le programme de l'agrégation et le programme de l'ADN — étaient eux-mêmes "agrégés ", comme le recto et le verso des pages sur lesquelles Derrida a tapé une bonne partie de son séminaire, avec son analyse de Jacob d'un côté et l'annonce d'un «meeting de mobilisation » pour le GREPH de l'autre.

Mais on peut aussi dire, en suivant l'autre versant de l'analyse de Derrida, que c'est grâce à cette loi de la contingence qui conditionne à la fois le programme génétique et le programme institutionnel, cette même loi d'une itérabilité générale, que le programme de l'agrégation, comme tout programme, est essentiellement ouvert à son extérieur, ouvert dans sa structure même à l'autre, ouvert donc à la variation, à la mutation, bref, à la nouveauté et à la surprise. Ce n'est donc peut-être pas par hasard — ou bien c'est tout à fait par hasard, grâce au hasard — si le même étudiant qui s'est fait "coller» à l'épreuve orale lors de ce fameux examen de l'agrégation aura pris un tel intérêt, une fois devenu professeur, à la notion de "programme» dans toutes ses manifestations ${ }^{29}$. Ce n'est sans doute pas plus un hasard si cet étudiant, une fois devenu professeur, aura procédé non pas à rejeter cette notion de programme mais à «négocier» avec elle afin de faire surgir du programme quelque chose d'imprévu, quelque chose qui lui échappe, quelque chose que l'on peut appeler un «événement». On peut dire que toute l'œuvre de Derrida — et toute sa pédagogie, à commencer par La vie la mort - en est la trace et la preuve.

28. Je ne vais pas plus loin dans cette direction puisque tout cela a été admirablement développé par Dawne McCance dans son livre Derrida's Life Death Seminar, New York, Fordham University Press, 2019. McCance montre en grand détail la façon dont Derrida dans son séminaire La vie la mort entrelace, comme les deux fils de l'ADN, le thème de la reproduction tel qu'il se trouve et dans la génétique et dans l'institution pédagogique dans le contexte de laquelle ce séminaire de Derrida avait lieu.

29. Derrida se livre à des réflexions sur cette douloureuse (mais finalement utile) expérience avec l'agrégation dans une discussion publique en 2003 avec Hélène Cixous; voir "Bâtons rompus ", dans Derrida d'ici, Derrida de là, Thomas Dutoit et Philippe Romanski (dir.), Paris, Galilée, 2009, p. 196-199. 